\title{
Tandem repeat recombinant proteins as potential antigens for the sero-diagnosis of Schistosoma mansoni infection
}

\author{
Yombo Dan Justin Kalenda a,b, Kentaro Kato a, Yasuyuki Goto ${ }^{c}$, Yoshito Fujii ${ }^{\mathrm{d}}$, Shinjiro Hamano ${ }^{\mathrm{a}, \mathrm{e}, *}$ \\ a Department of Parasitology, Institute of Tropical Medicine (NEKKEN), Nagasaki University, 1-12-4 Sakamoto, Nagasaki 852-8523, Japan \\ b Graduate School of Biomedical Sciences, Nagasaki University, 1-12-4 Sakamoto, Nagasaki 852-8523, Japan

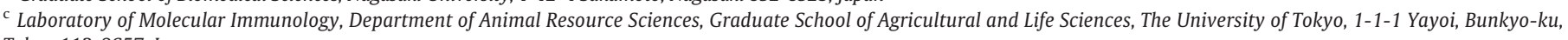 \\ Tokyo 113-8657, Japan \\ d Department of Eco-epidemiology, Institute of Tropical Medicine, Nagasaki University, 1-12-4 Sakamoto, Nagasaki 852-8523, Japan \\ e Nagasaki University Nairobi Research Station, NUITM-KEMRI Project, Nairobi, Kenya
}

\section{A R T I C L E I N F O}

\section{Article history:}

Received 21 April 2015

Received in revised form 15 June 2015

Accepted 22 June 2015

Available online 3 July 2015

\section{Keywords:}

Schistosoma mansoni

Sero-diagnosis

Tandem repeat

Antibodies

Animal model

\begin{abstract}
A B S T R A C T
The diagnosis of schistosome infection, followed by effective treatment and/or mass drug administration, is crucial to reduce the disease burden. Suitable diagnostic tests and field-applicable tools are required to sustain schistosomiasis control programs. We therefore assessed the potential of tandem repeat (TR) proteins for serodiagnosis of Schistosoma mansoni infection using an experimental mouse model.

TR genes in the genome of S. mansoni were searched in silico and 7 candidates, named SmTR1, 3, 8, 9, 10, 11 and 15, were selected. Total RNA was extracted from S. mansoni adult worms and eggs. Target TR genes were amplified, cloned, and the proteins were expressed in Escherichia coli competent cells. Female BALB/c mice were infected with $100 \mathrm{~S}$. mansoni cercariae and sera were collected each week post-infection for 18 weeks. The levels of IgG antibodies to SmTR antigens were compared to those to soluble egg antigen (SEA) and to soluble worm antigen preparation (SWAP). Sera of infected mice reacted to all the antigens whereas those of naïve mice did not. IgG responses to SmTR1, 3, 9 and 10 were detected at the early stage of infection. Interestingly, antibodies reacting to SmTR3, 9, 10 and 15 dramatically decreased 4 weeks after treatment with praziquantel, while those against SEA and SWAP remained elevated.

Our study suggests that TR proteins, especially SmTR10, may be suitable antigens for sero-diagnosis of infection by S. mansoni and are potential markers for monitoring and surveillance of schistosomiasis, including re-infection after treatment with praziquantel.
\end{abstract}

(C) 2015 Elsevier Ireland Ltd. All rights reserved.

\section{Introduction}

Schistosomiasis remains a public health problem as one of the major neglected tropical diseases. This helminthic disease is distributed worldwide and mainly affects resource-limited regions, with 74 countries reported to be endemic [1]. Over 230 million people are infected globally, with even more at risk of infection and complications [2]. Schistosomiasis is caused by the trematode, Schistosoma spp. In Africa, both Schistosoma mansoni (S. mansoni) and Schistosoma haematobium commonly co-exist, while the less-studied Schistosoma intercalatum is found in localized regions of central Africa. The Americas are affected by $S$. mansoni alone, while Asian schistosomiasis is caused by Schistosoma japonicum and Schistosoma mekongi [3].

\footnotetext{
* Corresponding author at: Department of Parasitology, Institute of Tropical Medicine (NEKKEN), Nagasaki University, 1-12-4 Sakamoto, Nagasaki 852-8523, Japan.

E-mail address: shinjiro@nagasaki-u.ac.jp (S. Hamano).
}

The pathogenesis of the disease is related to eggs that are produced in small vessels by adult female worms that have infected the body. In intestinal schistosomiasis, the eggs are trapped in tissues such as liver and intestines, whereas in uro-genital schistosomiasis (caused by S. haematobium), the eggs are trapped in the urinary bladder. These eggs are responsible for the formation of granuloma and subsequent associated complications such as hepatic fibrosis and hepatocellular carcinoma, chronic anemia, and stunted growth of chronically infected patients [3-5].

Early detection and treatment of the infection are crucial in preventing severe morbidities [6] and reducing the economic burden of the disease due to consecutive complications among affected populations $[7,8]$. Current schistosomiasis control and elimination programs rely mainly on mass drug administration (MDA) using praziquantel [9]. This isoquinoline derivative has significantly contributed to reducing both the morbidity and prevalence of schistosomiasis over the past several decades. However, praziquantel is ineffective on parasites in the juvenile stage $[10,11]$. Furthermore, 
reinfection by schistosomes is frequently observed in endemic areas, thus requiring repeated MDA activities in order to interrupt transmission and improve morbidity [12].

The commonly used standard assay for diagnosis of S. mansoni infection is the Kato Katz (KK) test [13] and involves the quantitative microscopic observation of parasite eggs in stool samples. It is currently acknowledged that the KK test lacks sensitivity especially in low endemic areas [14]. The search for a reliable tool for monitoring and surveillance of schistosomiasis has led to various antigens being investigated for sero-diagnostic tests [15]: schistosomula tegumental antigens [16,17], soluble egg antigens (SEA) [18], soluble worm antigen preparation (SWAP), and cercarial transformation fluid $[19,20]$. Tests based on the detection of specific antibodies against these antigens provide variable advantages and drawbacks that limit their applicability [21].

The detection of circulating cathodic antigen (CCA) in urine may be an alternative for monitoring and surveillance [22-24] and to date has provided consistent advantages in both lab and field studies. Although some field studies have reported a decreased sensitivity of the CCA test in low infection settings [25,26], it remains the alternative of choice to the KK test [22]. However, with the increasing widespread use of MDA and ongoing control programs, the diagnosis of schistosomiasis in low-endemic areas requires a test with high sensitivity and appropriate specificity. An immunodiagnostic test based on antibody detection has been shown to increase the detection efficiency of schistosomiasis especially in low-endemicity areas [27], and several countries such as China, Venezuela, and Brazil have introduced the antibody detectionbased test in their schistosomiasis elimination programs [28]. Although the differentiation of active from past infections has been challenging, the detection of specific antibody against SEA or SWAP has been used in laboratory and field settings to diagnose schistosome infection [29, 30]. Immunodiagnostic tests provide increased detection efficiency of schistosome infection in low-endemicity areas and among patients from non-endemic areas [28]. Recombinant antigens have been used as alternatives to SEA and SWAP in order to increase the specificity of the test and overcome the cross-reactions that occur when crude antigens are used; importantly, recombinant antigens are amenable to large-scale production. Thus, it is important to find suitable antigens for a sero-epidemiological approach to diagnose schistosome infection $[15,21,31]$.

Completion of the whole genome sequencing of S. mansoni [32] and the availability of increasingly sophisticated bio-informatics tools have provided potential resources in schistosome research for further screening of promising antigen targets useful either for diagnosis [33,34] or vaccination [35].

Tandem repeat (TR) genes have been reported to code polypeptides recognized by $\mathrm{B}$ cell receptors and have been studied in different parasites, including Plasmodium [36,37], Leishmania [38], Trypanosoma [39,40] and S. japonicum [41]. TR genes in S. mansoni have been studied throughout the life cycle of the parasite as modulators of gene transcription [42]. The TR non-coding genes Sm1-7 and Dra-1 have been used as markers of S. mansoni and S. haematobium infection, respectively, in intermediate host snails $[43,44]$. Sm1-7 is also used to diagnose S. mansoni human infections by detecting this highly repeated gene in biological samples such as stool, blood or urine $[25,45,46]$. The application of these techniques, however, requires the use of sophisticated tools such Real Time polymerase chain reaction (RT-PCR) or PCR equipment, restricting their field study application in endemic areas often found in resourcelimited countries.

TR antigens have been used to diagnose $S$. japonicum infection with interesting outcomes [41]. Here, we investigated the potential of TR antigens in a diagnostic test for $S$. mansoni infection in mice by developing an Enzyme-Linked Immunosorbent Assay (ELISA) to detect reactive immunoglobulin $\mathrm{G}(\operatorname{Ig} G)$ in the sera of infected mice.

\section{Materials and methods}

\subsection{Parasite}

A Puerto Rican strain of S. mansoni was used. The life cycle of this parasite is maintained in the animal facilities of Nagasaki University by passage through Biomphalaria glabrata snails and ICR mice or jirds. ICR mice were percutaneously infected with 250 cercariae, sacrificed 7 weeks after infection, and adult worms were collected from the portal vein by intra-cardiac perfusion with phosphate buffer saline (PBS). Eggs were isolated from the liver of infected mice as previously described [47, 48].

\subsection{Animals, infection and treatment}

Six week old female BALB/c mice were purchased from SLC (Shizuoka, Japan) and maintained in the animal facilities of Nagasaki University. These mice were kept in environmentally controlled, specific pathogenfree conditions, with free access to food and water. Animal housing, handling and feeding were done in compliance with the recommendations of Nagasaki University. All the experiments were approved by the ethical committee of Nagasaki University and were conducted following the animal facility guidelines.

Mice were percutaneously infected with $100 \mathrm{~S}$. mansoni cercariae in the inguinal area. At each time point, 6 mice were sacrificed and the liver and intestine were analyzed for the presence of eggs; adult worms were also collected through the portal vein by perfusion, starting 5 weeks post-infection.

A group of 6 mice were treated twice with praziquantel (Sigma, St. Louis, MO) at $300 \mathrm{mg} / \mathrm{kg}$ body weight 11 weeks post-infection with a 2 day interval [49]. The drug was dissolved in distilled water and $200 \mu \mathrm{l}$ of the solution was administrated by gavage; the control group of 6 mice received the equivalent volume of distilled water. One week post-treatment, 2 mice were sacrificed to confirm the absence of adult worms by portal vein perfusion, allowing verification of the efficacy of the treatment.

\subsection{Sera}

Blood was collected from mice 3 and 5 weeks post-infection, and also weekly from 5 weeks post-infection under anesthesia by periorbital puncture. Mice were sacrificed to confirm infection through the presence of worms and liver lesions. At each time point, 6 mice were sacrificed and submitted to perfusion of the hepatic portal system, as described elsewhere [47]. Sera were collected and aliquots were stored at $-30{ }^{\circ} \mathrm{C}$ until use. Sera collected from 6 uninfected mice were used as a negative control.

Sera were also collected from the treated group and their matched controls without sacrificing the mice at 11 weeks post-infection, before treatment, and 1, 5 and 7 weeks post-treatment (corresponding to 12 , 16 and 18 weeks post-infection, respectively).

\subsection{Preparation of SWAP and SEA crude proteins}

\subsubsection{Soluble worm antigen preparation (SWAP)}

Adult worms were harvested from mice infected with S. mansoni cercariae 7 weeks post-infection by portal vein perfusion; the worms were washed 3 times with PBS, homogenized using a mechanical grinder, and centrifuged at $10,000 \times g$ for $1 \mathrm{~h}$ at $4{ }^{\circ} \mathrm{C}$. The supernatant was collected and filtered through a $0.22 \mu \mathrm{m}$ mesh. The protein concentration was assessed using the Bradford method (Bio-Rad Laboratory, Hercules, $\mathrm{CA}$ ) and then the protein was aliquoted and stored at $-30^{\circ} \mathrm{C}$.

\subsubsection{S. mansoni soluble egg antigen (SEA)}

Soluble crude egg antigen (SEA) was prepared as previously reported [48]. Briefly, purified eggs from the livers of infected ICR mice were re- 
Table 1

Oligonucleotide primers used for the cloning of S. mansoni tandem repeat genes.

\begin{tabular}{|c|c|c|}
\hline Antigens & & Sequence \\
\hline \multirow[t]{2}{*}{ SmTR1 } & Fwd & 5'-GGATCCGATTACGGTGTATCTGGTGTGG-3' \\
\hline & Rev & 5'-AAGCTTCTAAGAAATTGTCTCTGATGACACAA-3' \\
\hline \multirow[t]{2}{*}{ SmTR3 } & Fwd & 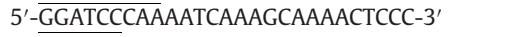 \\
\hline & Rev & 5'-AAGCTTCTACAAGTCCTGATGACTAGTCTCAG-3' \\
\hline \multirow[t]{2}{*}{ SmTR8 } & Fwd & $5^{\prime}-\overline{\text { CCAGGATCCACACGCTACAAT-3' }}$ \\
\hline & Rev & 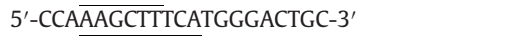 \\
\hline \multirow[t]{2}{*}{ SmTR9 } & Fwd & 5'-GGATCCTCCGACTCGGATTCGAGT-3' \\
\hline & Rev & 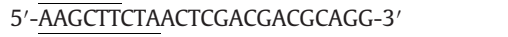 \\
\hline \multirow[t]{2}{*}{ SmTR10 } & Fwd & 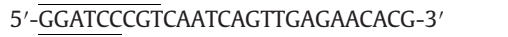 \\
\hline & Rev & 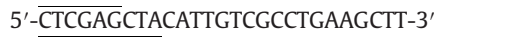 \\
\hline \multirow[t]{2}{*}{ SmTR11 } & Fwd & $5^{\prime}$-CCAGGATCCGAAATGCAGG-3' \\
\hline & Rev & 5'-GCCAAAGCTTTCAAACATGATG-3' \\
\hline \multirow[t]{2}{*}{ SmTR13 } & Fwd & 5'-GGATCCTCTATTAATTTGGGTGATGAAATACA-3' \\
\hline & Rev & 5'-AAGCTTCTATTCCATGTTTTCAGGATGTG-3' \\
\hline \multirow[t]{2}{*}{ SmTR15 } & Fwd & 5'-CCAGGATCCAAGAAGAAGACGAAA-3' \\
\hline & Rev & 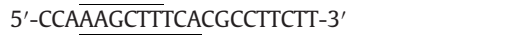 \\
\hline \multirow[t]{2}{*}{ Tubulin } & Fwd & 5'-TGG TGT CCA GGT TTC AA-3' \\
\hline & Rev & 5'-ACA AAT GCA CGT TTT GCG TA-3' \\
\hline
\end{tabular}

Underlines indicate the restriction enzyme sites inserted and dashed underlines indicate the stop codon.

suspended in $5 \mathrm{ml}$ ice-cold PBS at a concentration of 100,000 eggs $/ \mathrm{ml}$. The suspended eggs were sonicated for $15 \mathrm{~min}$ on ice to prevent heating until more than $95 \%$ of the eggs were destroyed. The sonicated eggs were centrifuged at $2000 \times \mathrm{g}$ for $20 \mathrm{~min}$ at $4{ }^{\circ} \mathrm{C}$. The supernatant was collected in a sterile tube, then ultracentrifuged at $100,000 \times g$ for $1 \mathrm{~h}$ at $4^{\circ} \mathrm{C}$. The supernatant was collected and the protein concentration was determined as for SWAP. SEA was aliquoted and stored at $-30^{\circ} \mathrm{C}$ until use.

\subsection{Cloning and expression of recombinant SmTR proteins}

S. mansoni total RNA was extracted from adult worms and eggs using the TriZol (Invitrogen, Madison, WI) protocol. Total RNAs were reverse transcribed into a single stranded cDNA using Takara PrimeScript RT reagent kit (Perfect Real Time, Takara Bio Inc., Otsu, Japan) following the manufacturer's protocol. Sequences encoding the TR domains of the selected genes were PCR-amplified using specific primers containing restriction enzyme ligation sites at both ends: BamHI at the $5^{\prime}$ end and HindIII at the 3' end of the antigens, except for SmTR10, in which XhoI
SmTR

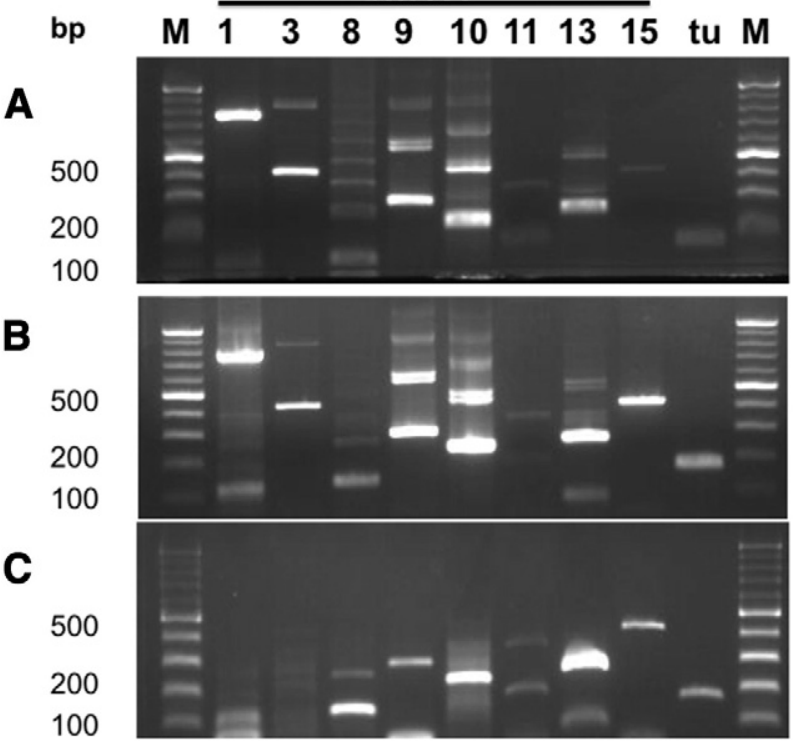

Fig. 1. PCR-gel electrophoresis of TR genes. PCR was conducted using an egg (A), adult worm (B), and cercaria (C) cDNA library as templates. 1:SmTR1, 3:SmTR3, 8:SmTR8, 9:SmTR9, 10:SmTR10, 11: SmTR11, 13: SmTR13 (TR positive control major tegmental Sm15), 15:SmTR15 and tu: tubulin, the internal control gene. M: marker for DNA size.

was inserted instead at the $3^{\prime}$ end. A stop codon was added on the $3^{\prime}$ end of each target gene sequence (Table 1 ). TR genes were amplified using Hot start Ex Taq DNA polymerase (Takara). S. mansoni alphatubulin gene was used as a constitutively expressed positive control gene to access the PCR reaction; specific primers targeting a 200 basepair (bp) length of alpha-tubulin were used for the reaction [50]. Using cDNA from both adult worms and eggs as PCR templates, the amplified TR gene domains were separated on 1.5\% agarose gel, the positive band corresponding to one or two periods of the tandem repeat were cut from the gel and purified using a QIAQuick gel extraction kit (Qiagen, Hombrechikon, Switzerland). The purified PCR products were cloned into pGEM-T Easy vector (Promega, Madison, WI) and sequenced using an ABI 3730 sequencer (AB Applied Biosystems, Tokyo,

Table 2

Top 20 tandem repeat genes within the whole S. mansoni genome

\begin{tabular}{|c|c|c|c|c|c|c|c|c|c|}
\hline Score & Gene ID & Gene product & $\begin{array}{l}\text { Length } \\
\text { (bp) }\end{array}$ & TR period size & $\begin{array}{l}\text { B cell } \\
\text { epitope }\end{array}$ & $\begin{array}{l}\text { TR protein } \\
\text { size }(\mathrm{kDa})\end{array}$ & $\begin{array}{l}\text { TR copy } \\
\text { number }\end{array}$ & Identity & \\
\hline 7990 & Smp_187350.1 & Hypothetical protein & 5634 & 795 & 2 & 33 & 5 & $79 \% S . j$ & SmTR1 \\
\hline 7767 & Smp_157380.1 & Hypothetical protein & 8235 & 1122 & 13 & & 3.5 & $39 \% S . j$ & SmTR2 \\
\hline 5691 & Smp_015190.1 & Serine-rich repeat protein, putative & 3873 & 429 & 4 & 19 & 6.8 & no & SmTR3 \\
\hline 5453 & Smp_038730.5 & Hypothetical protein & 4392 & 1023 & 9 & & 2.7 & $80 \%$ S.j & SmTR4 \\
\hline 4739 & Smp_182770.1 & Hypothetical protein & 3891 & 1023 & 9 & & 2.3 & & SmTR5 \\
\hline 4135 & Smp_038730.7 & Hypothetical protein & 3858 & 1023 & 9 & & 2 & & SmTR6 \\
\hline 3286 & Smp_094710.1 & Alstrom syndrome protein & 3828 & 81 & 1 & & 21.8 & no & SmTR7 \\
\hline 3014 & Smp_193700.1 & Hypothetical protein & 4071 & 123 & 1 & $6 \times 2$ & 13.8 & no & SmTR8 \\
\hline 2856 & Smp_110280.1 & Hypothetical protein & 2022 & 294 & 3 & 14 & 4.9 & $76 \%$ S.j, S.h & SmTR9 \\
\hline 2386 & Smp_073450.1 & Hypothetical protein & 11154 & 228 & 2 & 12 & 5.7 & $62 \%$ S.j, S.h & SmTR10 \\
\hline 2021 & Smp_027870.1 & Hypothetical protein & 4281 & 171 & 2 & $8 \times 2$ & 6.4 & no & SmTR11 \\
\hline 1931 & Smp_046690.1 & Ubiquitin (ribosomal protein L40), putative & 1140 & 450 & 1 & & 2.4 & $100 \%$ S.j & SmTR12 \\
\hline 1873 & Smp_156770.1 & Major tegumental antigen Sm15 & 3096 & 249 & 2 & 13 & 3.9 & $42 \% S . j$ & SmTR13 \\
\hline 1697 & Smp_192220.1 & Hypothetical protein. BCRA2 domain & 1845 & 120 & 1 & & 13.2 & no & SmTR14 \\
\hline 1647 & Smp_038730.2 & Hypothetical protein & 3780 & 411 & 4 & 17 & 2 & $80 \% S . j$ & SmTR15 \\
\hline 1353 & Smp_160340.1 & Translation initiation factor IF-2, putative & 792 & 8 & 0 & & 85.1 & no & $\begin{array}{l}\text { SmTR16 } \\
\text { pseudogene }\end{array}$ \\
\hline 1298 & Smp_160680.1 & Hypothetical protein & 4827 & 147 & 0 & & 4.6 & $63 \% S . j$ & SmTR17 \\
\hline 1232 & Smp_090930.5 & Hypothetical protein & 2019 & 255 & 3 & & 2.6 & no & SmTR18 \\
\hline 1127 & Smp_158930.1 & Hypothetical protein & 4200 & 201 & 1 & & 2.9 & $68 \% S . j$ & \\
\hline 1000 & Smp_179670.1 & Hypothetical protein & 543 & 117 & 1 & & 4.3 & no & $\begin{array}{l}\text { SmTR20 } \\
\text { pseudogene }\end{array}$ \\
\hline
\end{tabular}

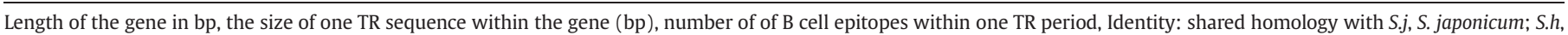

S. haematobium; and no, no homology with any species. The expressed proteins in this study are marked bold character. 
SmTR

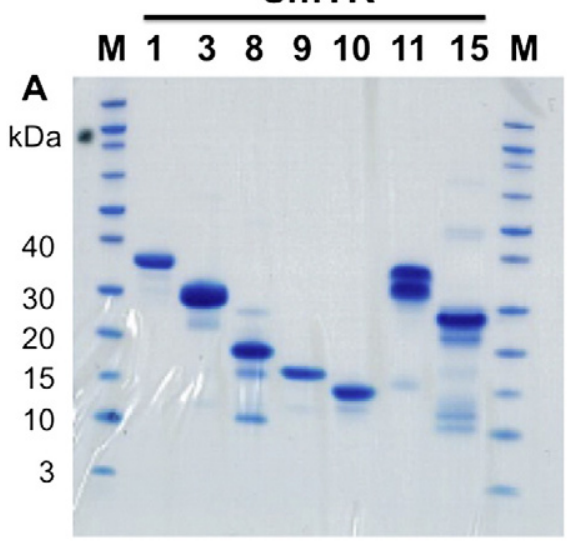

SmTR

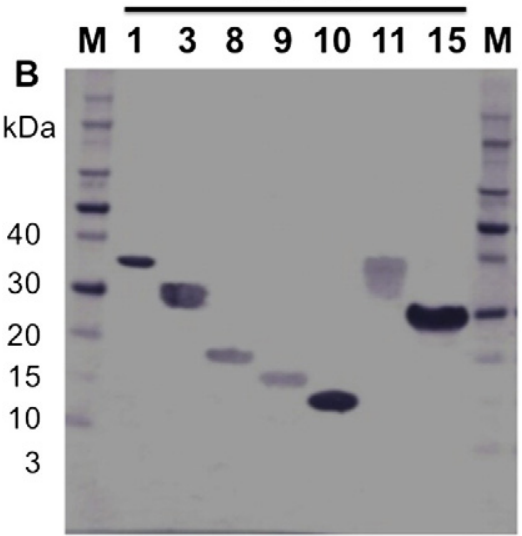

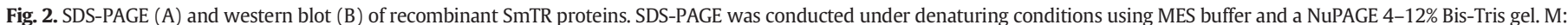

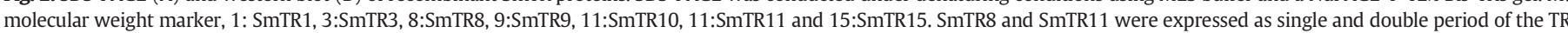
genes, thus SmTR8 presented 2 bands at 10 and $16 \mathrm{kDa}$, while SmTR11 showed a faint band at $16 \mathrm{kDa}$ and a strong dimer at $30 \mathrm{kDa}$.

Japan). The cloned fragments were excised and sub-cloned into pET28a vector (Novagen, Merck Chemicals, Darmstadt, Germany), then transformed into E. coli BL21 (DE3). Positive colonies of E. coli BL21 (DE3) competent cells were large-scale cultured in $200 \mathrm{ml}$ of $2 \times$ YT medium containing $100 \mu \mathrm{g} / \mathrm{ml}$ kanamycin (Sigma) at $37{ }^{\circ} \mathrm{C}$. SmTR protein expression was induced by adding $500 \mu \mathrm{l} 0.1 \mathrm{M}$ Isopropyl $\beta$-D-1-thiogalactopyranoside IPTG (Takara) and the culture was kept for $3 \mathrm{~h}$ at $37^{\circ} \mathrm{C}$. The pellet was stored at $-80^{\circ} \mathrm{C}$ until use. Extraction and purification of the target proteins were completed by Nickel-nitrilotriacetic acid Ni-NTA agarose (Qiagen) purification of the pellet under denaturing
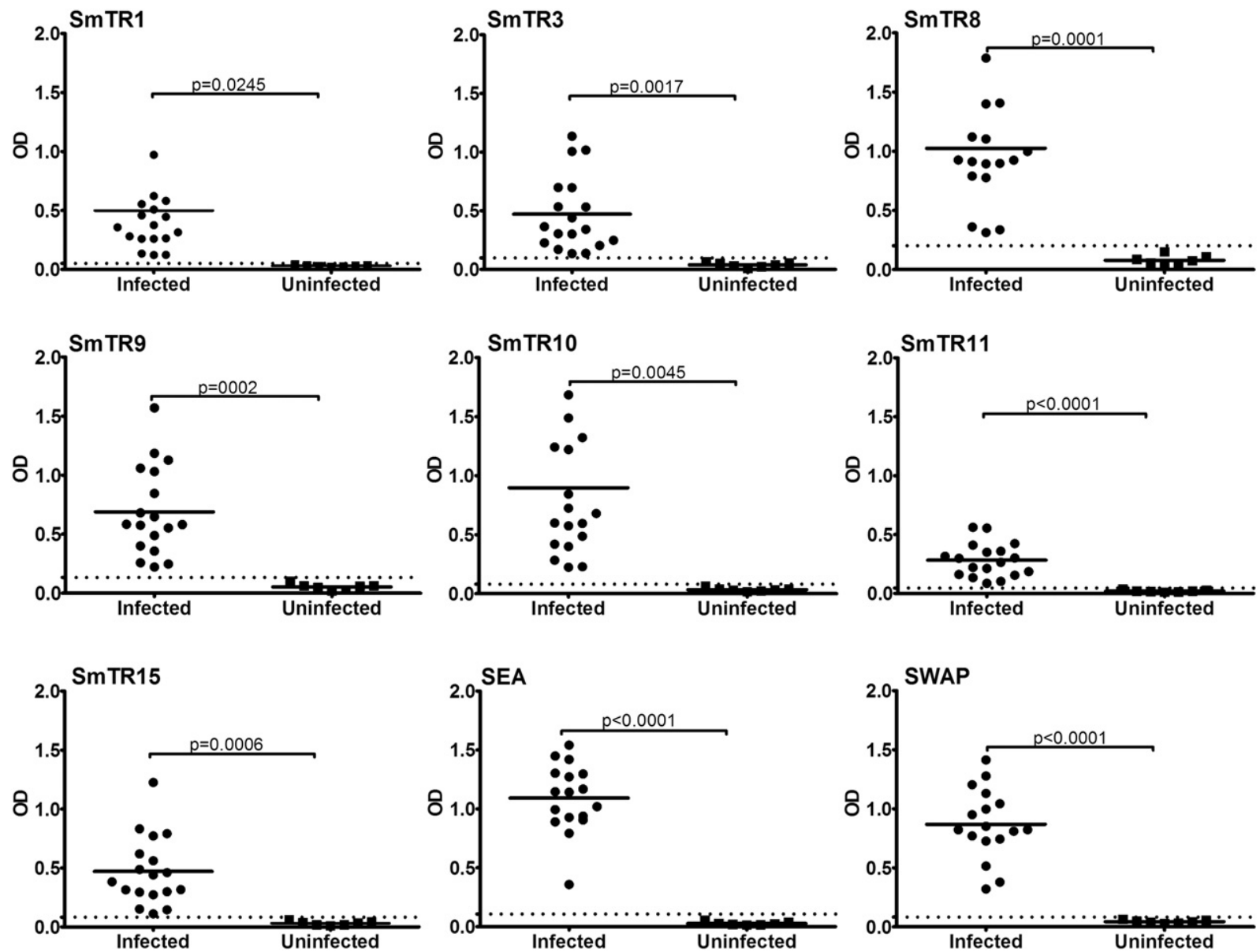

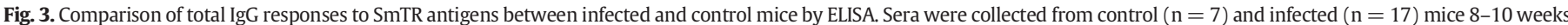

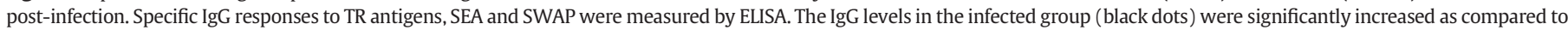

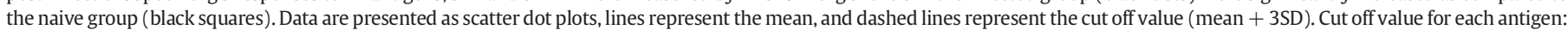
SmTR1, 0.052; SmTR3, 0.097; SmTR8, 0.200; SmTR9, 0.130; SmTR10, 0.081; SmTR11, 0.045; SmTR15, 0.083; SEA, 0.107 and SWAP, 0.08. $p$ value is indicated for each antigen. 
conditions, following the manufacturer's instructions. The extraction and purification of SmTR8 and 15 followed a slightly different procedure. Instead of lysing of $E$. coli culture pellet with $8 \mathrm{M}$ urea, Bugbuster protein extraction reagent (Novagen) was used following the manufacturer's protocol as it provided better purification conditions for soluble SmTR8 and 15. Expressed proteins were eluted from the column with $150 \mathrm{mM}$ imidazole. The purified proteins were dialyzed against $20 \mathrm{mM}$ Tris- $\mathrm{HCl}$ ( $\mathrm{pH}$ 8.5), then the buffer was exchanged with PBS using Amicon ultra centrifugal filters 3000 MWCO (Millipore,Volketswil, Switzerland).

The integrity of the proteins was assessed by polyacrylamide gel electrophoresis (SDS-PAGE) using 4-12\% NuPAGE ${ }^{\circledR}$ Novex Bis-Tris mini gels (Invitrogen, Carlsbad, CA) under denaturing and reducing conditions. The developed gels were stained with Coomassie blue using Simply Blue Safestain (Invitrogen).

In addition, western blotting was conducted using mouse anti-His tag monoclonal antibody (Novagen). Where the size of the recombinant protein did not correspond to the expected size on SDS-PAGE, the peptide sequence was analyzed by trypsin digestion and liquid chromatography-mass spectrometry (LC-MS/MS) following the detailed protocol described elsewhere [51]. After measuring the concentration of the recombinant proteins as described above for SWAP and SEA, the antigens were aliquoted and stored below $-30^{\circ} \mathrm{C}$.

\subsection{Antibody detection using ELISA}

Nunc MaxiSorp microtiter plates (Nalgene Nunc Int., Roskilde, Denmark) were coated with $100 \mu \mathrm{l}$ of $2 \mu \mathrm{g} / \mathrm{ml}$ (200 ng per well) recombinant SmTR antigen in coating buffer $\left(15 \mathrm{mM} \mathrm{Na}_{2} \mathrm{CO}_{3}, 35 \mathrm{mM} \mathrm{NaHCO}_{3}\right.$, $\mathrm{pH}$ 9.6), or $200 \mathrm{ng} / \mathrm{ml}$ for SEA and SWAP. Plates were then blocked with $1 \%$ bovine serum albumin (BSA) (Sigma) in PBS, pH 7.4 for $2 \mathrm{~h}$ at RT. Serum samples were diluted 400 times in PBST containing $0.1 \%$ BSA, added to the plates, and incubated at RT for $60 \mathrm{~min}$. Horseradish peroxidase-conjugated goat anti-mouse IgG antibody (R\&D, Minneapolis, MN) was used as secondary antibody at a dilution of 1:2000 in PBST containing $0.1 \% \mathrm{BSA}$, and tetramethylbenzidine (TMB) (BD Pharmingen, Allschwil, Switzerland) was added as substrate. Absorbance was measured at $450 \mathrm{~nm}$ using a Multiskan FC microplate reader (Thermo Scientific). All sera were assayed in duplicate. Sera collected from infected mice 8 and 9 weeks post-infection were pooled and considered as the control standard to assess the ELISA conditions; control negative sera were also included on the ELISA plate.

Specific $\operatorname{IgG}$ subtypes and IgE antibodies were detected using 50 times diluted sera of infected mice collected 9 weeks post-infection, and rat anti-mouse IgE-HRP-conjugated antibody (AbD Serotec, BioRad) or rat anti-mouse IgG subtype (1, 2a, 2b or 3$)$-HRP antibody
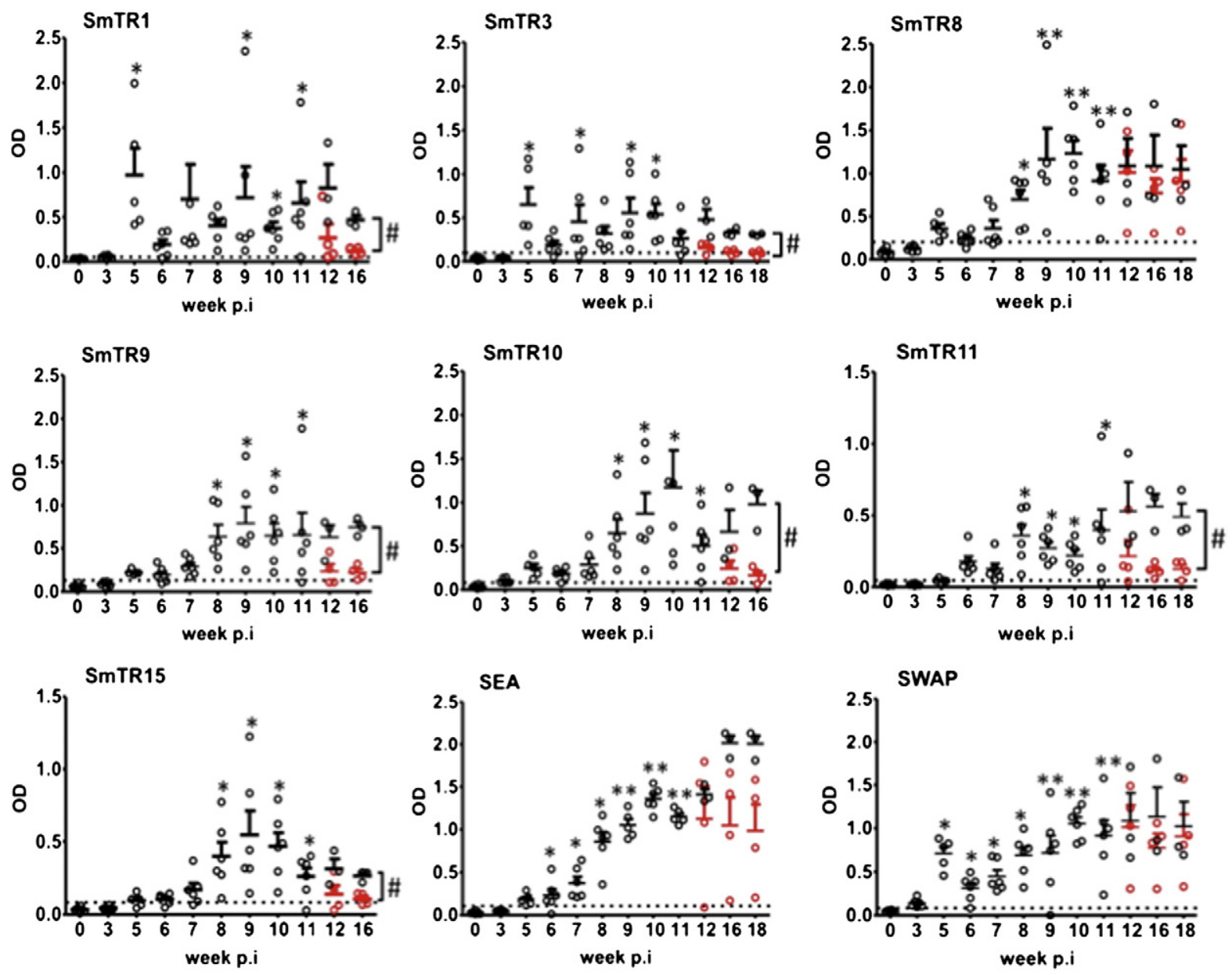

- Non treated

- Treated

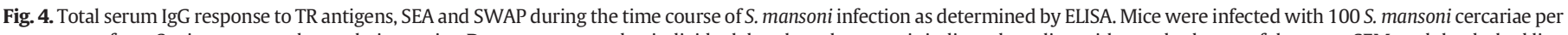

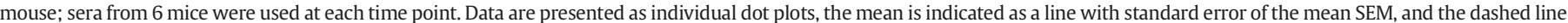

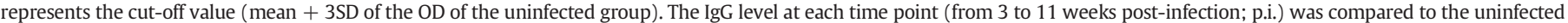

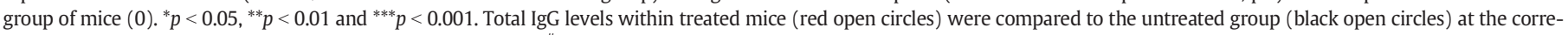
sponding time point after treatment (12,16 and 18 weeks p.i.), ${ }^{\#} p<0.05$. Data are representative of at least 3 independent experiments. 
(Invitrogen) were used at 10,000 dilution; the remaining ELISA steps were as described for total IgG detection.

\subsection{Statistical analysis}

The statistical software Graphpad Prism 5.0 (GraphPad Software Inc., La Jolla, CA) and Excel 2011 (Microsoft Corporation, Seattle, WA) were used for statistical analysis and graph generation. The cutoff point between infected and uninfected group results was estimated as the mean plus 3 standard deviation (mean $+3 \mathrm{SD}$ ) of the OD of the control group sera for each respective antigen. ANOVA and $t$ test were used for normally distributed samples, and the Mann-Whitney test or Kruskal-Willis test were used for all other samples. Linear regression was used to evaluate the diagnostic performance between TR antigens and SEA or SWAP. To compare the difference between groups, $\mathrm{p}<0.05$ was considered significant.

\section{Results}

\subsection{In silico analysis for the identification of tandem repeat genes}

The whole genome of S. mansoni was obtained from GeneDB [52] and screened to detect tandem repeat genes by in silico analysis using "Tandem repeats finder software" [53] following the scoring setting as previously described [38]. Out of 11,809 putative genes of this parasite, the top 20 genes containing TRs with a score equal to or greater than 1000 were selected for further analysis. These genes were named SmTR1 to 20, numbered in descending order of their TR score for easy use and recognition (Table 2). Most of these genes were annotated as coding genes for hypothetical proteins that, to our knowledge, have not yet been described, except for the Smp_156770.1 gene (SmTR13) coding for the major tegmental antigen Sm15 [54,55]. These 20 genes likely possess B cell epitopes in the TR region and were checked accordingly using the BCPRED epitope prediction program (http://ailab.ist.psu. edu/bcpred/predict.html). The predicted number of B cell epitopes are listed in Table 2.

The majority of TR genes were found to share homology with S. japonicum. There was less concern regarding cross-reactivity of S. mansoni and S. japonicum since their geographic distributions do not overlap. Although SmTR9 and 10 share some degree of homology with S. haematobium and S. japonicum, they were selected for further cloning and expression.

SmTR16 and the 20 genes were not amplified using their specific primers and thus were considered as pseudogenes. Antigens showing some homology with the mouse or human proteins were excluded from the study to prevent cross-reaction with the host antigens. The Smp_046690.1 (SmTR12) gene codes for ubiquitin, ribosomal protein L40. This gene shares similarities with genes conserved in various species, including S. japonicum and mouse. Similarly, the Smp_160680.1 (SmTR17) gene contains a sequence coding for a domain of breast cancer related protein type 2 (BCRA2) and thus was excluded from this study. The sequences of the Smp_038730.5 (SmTR4), Smp_182770.1 (SmTR5), Smp_038730.7 (SmTR6) and Smp_038730.2 (SmTR15) genes share similarity in the TR period sequences, suggesting that these different genes code for the same TR protein. Therefore, the gene coding the shortest TR period, SmTR15, was selected and expressed as a representative protein. SmTR2 was excluded from the study since the length of the TR period exceeded $1000 \mathrm{bp}$, the maximum size set for our study. The major tegumental protein Sm15 (SmTR13) has been reported to play a role in the protective immunity and evasion mechanism of S. mansoni $[54,55]$. Since our interest was to screen new potential diagnostic antigens, this gene was only used to confirm the amplification of the TR gene and was subsequently excluded from the protein expression study.

Thus, only 7 SmTR genes were considered for protein expression.

\subsection{Cloning and expression of SmTR antigens}

SmTR1 and 3 were dominantly expressed in S. mansoni at the adult and egg stage (Fig. 1A\&B) and were not expressed in the cercaria stage (Fig. 1C). The remaining SmTR genes were expressed at all stages of the parasite life cycle within the host; nevertheless, the expression level of SmTR11 was weak as compared to that of tubulin and other SmTR genes. SmTR8 showed a different expression pattern in adult, egg and cercaria; suggesting that it may have some functional role in the life cycle of the parasite. The recombinant proteins were expressed as a single TR period in the gene; the exceptions were SmTR8 and SmTR11, with TR period sizes of 123 and 171, respectively, which were expressed as both single and double TR periods.

The purity and integrity of the antigens were assessed by SDS-PAGE and western blotting analysis (Fig. 2A\&B). SmTR3 annotated as a putative serine-rich repeat protein and had a mass of $30 \mathrm{kDa}$, larger than the expected mass $19 \mathrm{kDa}$. The sequence of this protein was confirmed by mass spectrometry analysis after trypsin digestion (Fig. S1). Similarly, SmTR15 migrated less than expected. Most SmTR8 and SmTR11 proteins were detected as dimers on SDS-PAGE whereas only the dimeric bands were observed on the western blotted membrane. These results confirmed the expression of the target proteins (Fig. 2).

\subsection{Antibody response to TR antigens}

To evaluate the potential of these antigens to induce antibody responses, sera were collected from naïve and infected mice 8 to 10 weeks post-infection and were subjected to ELISA probing for total IgG. The sera from infected mice reacted to each antigen, but not the
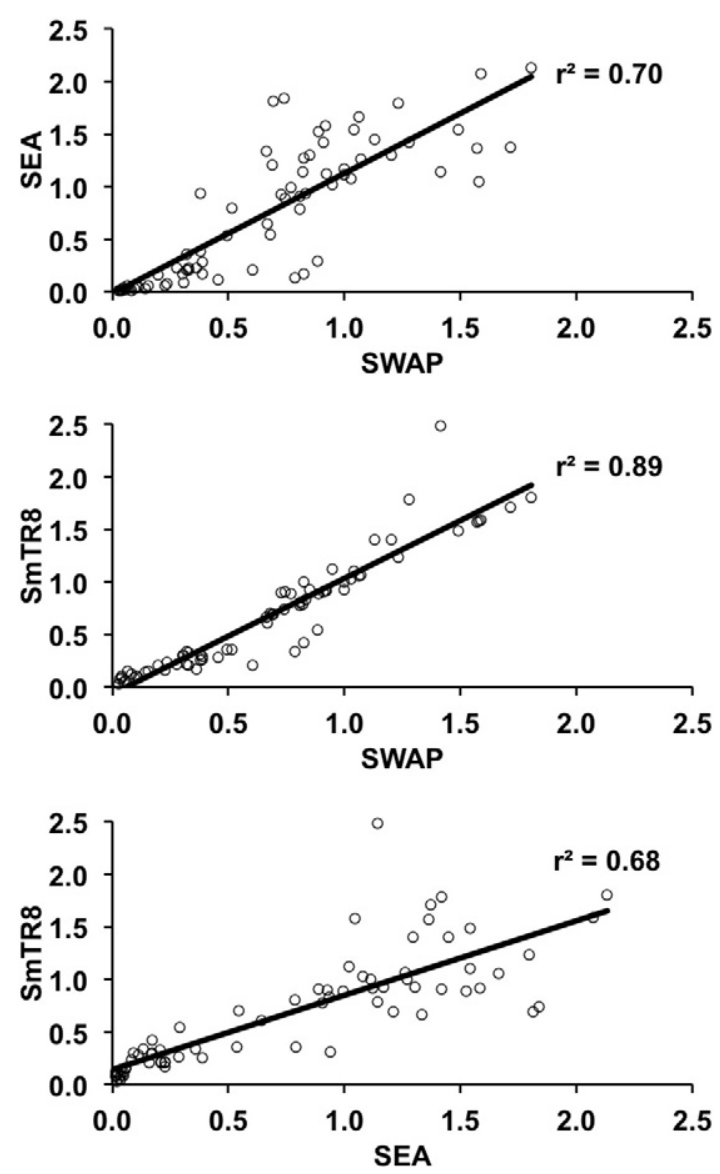

Fig. 5. Correlation between IgG responses to SmTR8 antigen, SEA and SWAP. The OD values of IgG responses to $S$. mansoni antigens were assessed using concordance with SEA and SWAP as reference; $\mathrm{r}^{2}$ : correlation coefficient value. 
sera from naïve mice. These results were consistent with those obtained for SEA and SWAP (Fig. 3).

As the intensity and expression pattern of some SmTR genes is distinct between the egg, cercaria and adult worm stages of the parasite life cycle (Fig. 1), we measured the reaction of $\operatorname{Ig} G$ antibodies to TR antigens during the course of infection. Significant IgG reaction to crude SWAP was detected in sera 5 weeks post-infection compared to the cut-off value, while IgG responses to SEA increased from 6 weeks post-infection. IgG levels to both SWAP and SEA peaked at 10 weeks post-infection (Fig. 4). IgG to SmTR antigens was detected in sera 5 weeks post-infection, similar to SWAP IgG (Fig. 4). The increase in the antibody to SmTR15 was delayed to 7 weeks postinfection. SmTR1, 8 and 10 induced a higher level of IgG antibodies compared to other recombinant antigens. The IgG response to SmTR10 increased by as early as 3 weeks post-infection when compared with the cut-off value. Nevertheless, a statistically significant increase in IgG antibodies to recombinant antigens could be detected only from either 8 or 9 weeks post-infection when compared to $\operatorname{IgG}$ levels in naïve sera. Unlike other SmTR antigens, total IgG levels to SmTR1 and 3 were exceptionally and significantly elevated at 5 weeks post infection.

\subsection{Antibody response after praziquantel treatment}

A general characteristic of antibody responses is that their level is maintained in individuals for a certain period of time even after clearance of external antigens. We therefore assessed the levels of IgG response to each SmTR antigen, as well as those to SEA and SWAP, after treatment of infected mice with praziquantel. IgG levels to SEA and SWAP remained high, comparable to those in untreated mice (Fig. 4). Interestingly, antibodies to the recombinant antigens tended to rapidly decrease after treatment with praziquantel; the exception was SmTR8, for which the IgG levels remained high, similar to those for SWAP. The response to SmTR3, 10 and 15 dramatically decreased to baseline level after treatment (Fig. 4).
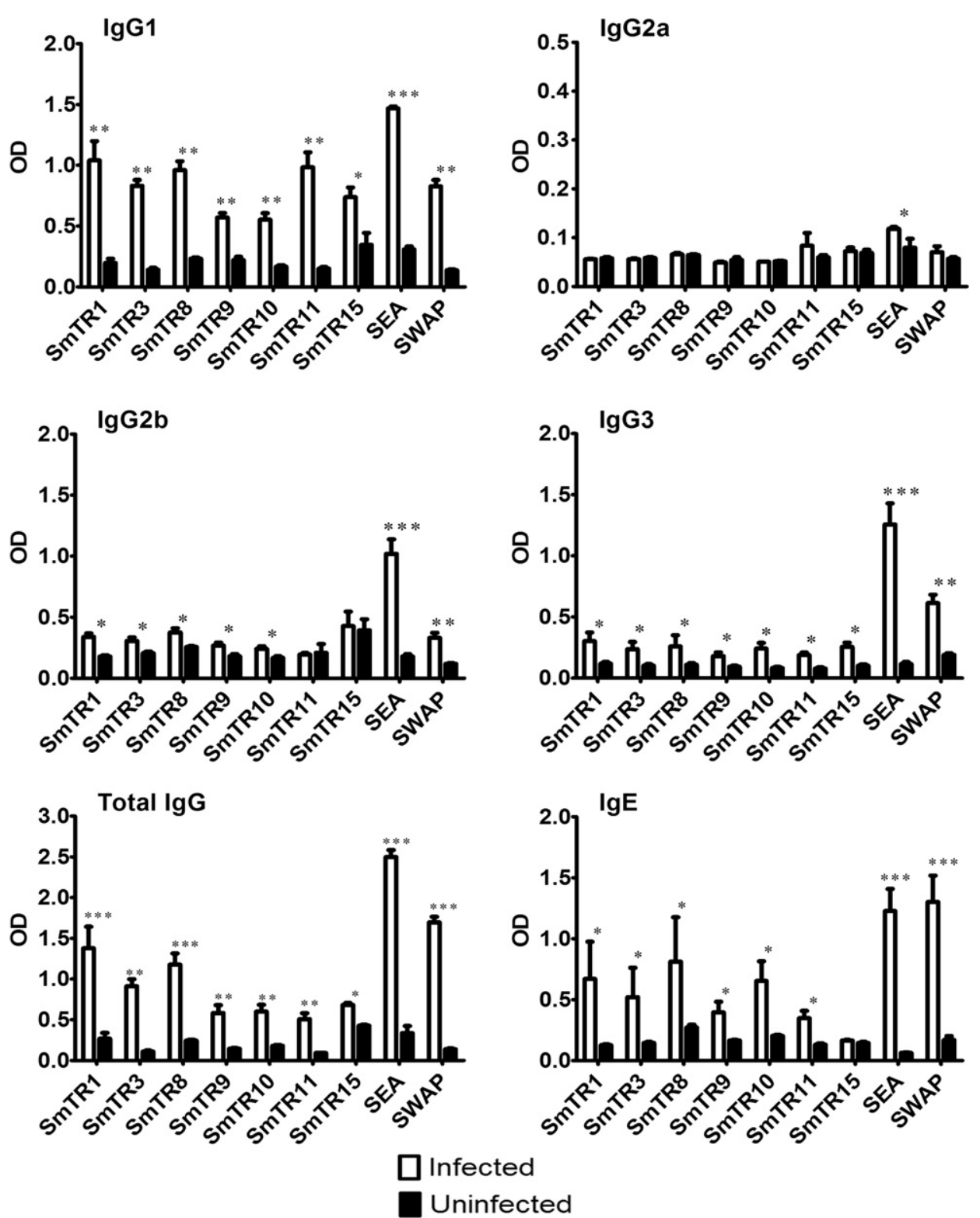

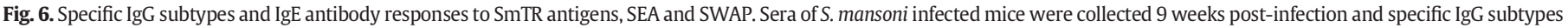

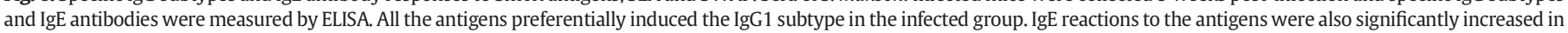
the infected group, except for SmTR15. Data are presented as mean + SEM. ${ }^{*} p<0.05,{ }^{* *} p<0.01$ and ${ }^{* * *} p<0.001$. Data are representative of 2 independent experiments. 


\subsection{Correlation between SmTRs, SEA and SWAP}

The diagnostic performance of SEA showed a close correlation with SWAP (Fig. 5), similar to previously reported studies [56]. Among the SmTR antigens, SmTR8 showed the highest correlation with SWAP and SEA (Figs. 5 and S2A \& B). However, antibodies to SmTR8 remained increased even after treatment with praziquantel, unlike for SmTR3, 9 and 10 (Fig. 4). SmTR1 and 3 showed the lowest correlation with both SEA and SWAP (Fig. S2A \& B).

\subsection{Antibody subtype specific reaction}

IgGs have different subtypes in human and mouse, with different functions [57]. IgG4 subtypes in human and IgG1 in mouse are involved in resistance and susceptibility to schistosomiasis [58,59] and thus are of great interest for the diagnosis of helminthic diseases [60].

We accordingly attempted to analyze the IgG subtypes raised against these antigens, as well as detecting specific IgE antibody, an important immunoglobulin during helminthic infections, including schistosomiasis [59]. The IgG1 subtype was found to be the dominant IgG response to the recombinant antigens (Fig. 6), with IgG1 to SmTR1 and 8 being present at the highest level. Specific IgG3 and IgG2b were also increased respectively after IgG1. In addition, there was no real increase of IgG2a response to SmTR antigens; this IgG subtype was only detected against SEA.

Specific IgE responses to SmTR antigens were significantly increased in the infected group, with the exception of IgE responses to SmTR15, which were not induced after infection.

\section{Discussion}

The main purpose of our study was to evaluate S. mansoni TR recombinant proteins as potential candidates for the sero-epidemiology of schistosomiasis. All antigens successfully expressed in this study were previously reported as hypothetical or putative proteins [32]. Our results provided further evidence of their expression at the protein level during the life cycle of the $S$. mansoni parasite. These recombinant TR antigens induced antibody responses that were detected during S. mansoni infection. Antibody reaction to SmTR10 was detected from 3 weeks post-infection onwards as compared to the cut off value, much earlier than those to SEA, which are significantly positive 6 weeks post-infection (Fig. 4), corresponding to the release of eggs by the adult female [5]. In addition, the level of specific IgG antibodies to SmTR1, 3, 9, 10 and 15 rapidly decreased after treatment with praziquantel, suggesting that these antigens could be promising candidates not only for the diagnosis of S. mansoni infection, but also as potential markers in monitoring re-infection after treatment with praziquantel.

The expression pattern of the SmTR8 gene was distinct in egg, adult worm and cercaria (Fig. 1), suggesting that this gene might have a function within the life cycle of schistosome. Further studies are required to assess the function of this antigen.

Moreover, specific IgG responses to SmTR8 showed a strong correlation with both SEA and SWAP in diagnostic performance. SEA preparation requires a consistent volume of eggs and animals [48] that are time and resources consuming, thus SmTR8 might be an alternative candidate diagnostic test to SEA and SWAP, since these crude antigens are commonly used in non endemic countries and laboratory settings to diagnose schistosome infection [18,30].

Despite the limitations of antibody detection-based diagnosis in discriminating current from past infection, its high sensitivity provides advantages for detecting schistosomiasis cases in low-endemic areas [16, 20].

The size discrepancy of SmTR3 on SDS-PAGE (Fig. 2) might be explained by the serine-rich characteristics of this antigen that induced a slow migration on the gel. A similar condition was described with histidine-rich protein-2 used for the rapid diagnosis of Plasmodium falciparum infection $[61,62]$, with the serine-rich repeat invariant surface glycoprotein ISG of Trypanosoma brucei [63], and with the glycoproteins p120 and p140 of the bacteria Ehrlichia chaffeensis and Ehrlichia canis [64].

Most specific antibody responses to TR antigens were of the IgG1 subtype and several of these antigens induced an increase in specific IgE antibodies in sera (Fig. 6). As IgG4 in humans decreases dramatically after treatment with praziquantel, detection of IgG4 antibody responses against helminthic infections can help differentiate active infection from prior exposure [60]. Thus, further study of human schistosomiasis will be required with the aim of detecting specific IgG4 to SmTR antigens in sera and urine, thereby providing more insights into these currently hypothetical proteins. At 5 to 10 weeks after infection with 100 cercariae, the median number of worms collected from BALB/c mice was 30 with interquartile range of 15 . We could not find any correlation between the number of worm and the IgG antibody levels in this condition. Although BALB/c mice can tolerate more cercariae than some other mouse strains, 100 is a lot for any mouse. In order to confirm the reproducibility of our data, we infected BALB/c and C57BL/6 mice with 50 cercariae per mouse in a different experiment, and the outcomes obtained were comparable with the present experiments (data not shown).

In silico screening of the TR genes showed shared similarities among Schistosoma species, implying potential cross-reaction in co-endemic areas, especially with $S$. haematobium in sub-Saharan regions where many foci of co-infection are reported. It could be considered as an advantage to detect different species with one diagnostic test in field settings $[20,65]$, because the treatment is the same for infection by these schistosome species.

The main purpose of this study was to identify and express TRcontaining antigens of $S$. mansoni and focused on a mouse model using a limited number of mice; consequently, this study could not estimate the exact sensitivity and specificity of the proposed diagnostic antigen tests.

\section{Conclusions}

The availability of suitable markers for monitoring schistosome infections is urgently required for the control and elimination of schistosomiasis. SmTR antigens, and especially SmTR10, are potential markers for the diagnosis of infection, and for monitoring re-infection by S. mansoni after treatment with praziquantel.

Since the goal is to use these antigen candidates for identifying human infection cases, our future work will evaluate the validity of the proposed antigen candidates using human samples from a region endemic for schistosomiasis.

Supplementary data to this article can be found online at http://dx. doi.org/10.1016/j.parint.2015.06.012.

\section{Acknowledgments}

Leo Uchida helped to analyze the mass spectrometry peptide sequence of the SmTR antigens. We thank the members of the Department of Parasitology, Institute of Tropical Medicine, Nagasaki University, for the assistance and support during the study. YDJK was supported by the Japanese government through the Ministry of Education, Sport and Technology (MEXT) fellowship. This work was partially supported by Strategic Young Researcher Overseas Visits Program for Accelerating Brain Circulation 2013-2015 (S2509 to S.H.) and the Cooperative Research Station of Tropical Medicine (NEKKEN), Nagasaki University.

\section{References}

[1] L. Chitsulo, D. Engels, A. Montresor, L. Savioli, The global status of schistosomiasis and its control, Acta Trop. 77 (2000) 41-51. 
[2] D.G. Colley, A.L. Bustinduy, W.E. Secor, C.H. King, Human schistosomiasis, Lancet 383 (2014) 2253-2264

[3] B. Gryseels, K. Polman, J. Clerinx, L. Kestens, Human schistosomiasis, Lancet 368 (2006) 1106-1118.

[4] B. Gryseels, Schistosomiasis, Infect. Dis. Clin. N. Am. 26 (2012) 383-397.

[5] E.J. Pearce, A.S. MacDonald, The immunobiology of schistosomiasis, Nat. Rev. Immunol. 2 (2002) 499-511.

[6] D. Gurarie, X. Wang, A.L. Bustinduy, C.H. King, Modeling the effect of chronic schistosomiasis on childhood development and the potential for catch-up growth with different drug treatment strategies promoted for control of endemic schistosomiasis, Am. J. Trop. Med. Hyg. 84 (2011) 773-781.

[7] P.J. Hotez, A. Kamath, Neglected tropical diseases in sub-Saharan Africa: review of their prevalence, distribution, and disease burden, PLoS Negl. Trop. Dis. 3 (2009) e412.

[8] C.H. King, Parasites and poverty: the case of schistosomiasis, Acta Trop. 113 (2010) 95-104.

[9] Committee WHOE, Prevention and control of schistosomiasis and soil-transmitted helminthiasis, World Health Organ. Tech. Rep. Ser. 912 (2002) i-vi (1-57, back cover).

[10] R. Gonnert, P. Andrews, Praziquantel, a new broad-spectrum antischistosomal agent, Z. Parasitenkd. 52 (1977) 129-150.

[11] M.J. Doenhoff, D. Cioli, J. Utzinger, Praziquantel: mechanisms of action, resistance and new derivatives for schistosomiasis, Curr. Opin. Infect. Dis. 21 (2008) 659-667.

[12] S.M. Njenga, F.M. Mutungi, C.N. Wamae, M.T. Mwanje, K.K. Njiru, et al., Once a year school-based deworming with praziquantel and albendazole combination may not be adequate for control of urogenital schistosomiasis and hookworm infection in Matuga District, Kwale County, Kenya, Parasit. Vectors 7 (2014) 74

[13] N. Katz, A. Chaves, J. Pellegrino, A simple device for quantitative stool thick-smear technique in Schistosomiasis mansoni, Rev. Inst. Med. Trop. Sao Paulo 14 (1972) 397-400.

[14] A. Kongs, G. Marks, P. Verle, P. Van der Stuyft, The unreliability of the Kato-Katz technique limits its usefulness for evaluating $S$. mansoni infections, Trop. Med. Int. Health 6 (2001) 163-169.

[15] M.G. Cavalcanti, L.F. Silva, R.H. Peralta, M.G. Barreto, J.M. Peralta, Schistosomiasis in areas of low endemicity: a new era in diagnosis, Trends Parasitol. 29 (2013) 75-82.

[16] R. Grenfell, W. Martins, V. Silva-Moraes, N. Araujo, E. Oliveira, et al., The schistosomula tegument antigen as a potential candidate for the early serological diagnosis of schistosomiasis mansoni, Rev. Inst. Med. Trop. Sao Paulo 55 (2013) 75-78.

[17] R.F. Grenfell, W. Martins, S.C. Drummond, C.M. Antunes, I. Voieta, et al., Acute schistosomiasis diagnosis: a new tool for the diagnosis of schistosomiasis in a group of travelers recently infected in a new focus of Schistosoma mansoni, Rev. Soc. Bras. Med. Trop. 46 (2013) 208-213.

[18] T. Van Gool, H. Vetter, T. Vervoort, M.J. Doenhoff, J. Wetsteyn, et al., Serodiagnosis of imported schistosomiasis by a combination of a commercial indirect hemagglutination test with Schistosoma mansoni adult worm antigens and an enzyme-linked immunosorbent assay with S. mansoni egg antigens, J. Clin. Microbiol. 40 (2002) 3432-3437.

[19] D. El Aswad Bel, M.J. Doenhoff, A.S. El Hadidi, W.J. Schwaeble, N.J. Lynch, Use of recombinant calreticulin and cercarial transformation fluid (CTF) in the serodiagnosis of Schistosoma mansoni, Immunobiology 216 (2011) 379-385.

[20] N. Nausch, E.M. Dawson, N. Midzi, T. Mduluza, F. Mutapi, et al., Field evaluation of a new antibody-based diagnostic for Schistosoma haematobium and S. mansoni at the point-of-care in northeast Zimbabwe, BMC Infect. Dis. 14 (2014) 165.

[21] L.I. Gomes, M.J. Enk, A. Rabello, Diagnosing schistosomiasis: where are we? Rev. Soc Bras. Med. Trop. 47 (2014) 3-11.

[22] D.G. Colley, S. Binder, C. Campbell, C.H. King L.A. Tchuem Tchuente, et al., A fivecountry evaluation of a point-of-care circulating cathodic antigen urine assay for the prevalence of Schistosoma mansoni, Am. J. Trop. Med. Hyg. 88 (2013) 426-432.

[23] I.S. Barsoum, K.A. Kamal, S. Bassily, A.M. Deelder, D.G. Colley, Diagnosis of human schistosomiasis by detection of circulating cathodic antigen with a monoclonal antibody, J. Infect. Dis. 164 (1991) 1010-1013.

[24] H.L. Shane, J.R. Verani, B. Abudho, S.P. Montgomery, A.J. Blackstock, et al., Evaluation of urine CCA assays for detection of Schistosoma mansoni infection in Western Kenya, PLoS Negl. Trop. Dis. 5 (2011) e951.

[25] N. Lodh, J.C. Mwansa, M.M. Mutengo, C.J. Shiff, Diagnosis of Schistosoma manson without the stool: comparison of three diagnostic tests to detect Schistosom [corrected] mansoni infection from filtered urine in Zambia, Am. J. Trop. Med. Hyg 89 (2013) 46-50.

[26] M. Legesse, B. Erko, Field-based evaluation of a reagent strip test for diagnosis of schistosomiasis mansoni by detecting circulating cathodic antigen (CCA) in urine in low endemic area in Ethiopia, Parasite 15 (2008) 151-155.

[27] B.A. de Noya, O. Noya, C. Balzan, I.M. Cesari, New approaches for the control and eradication of schistosomiasis in Venezuela, Mem. Inst. Oswaldo Cruz 87 (Suppl. 4) (1992) 227-231.

[28] M.C. Carvalho do Espirito-Santo, P.L. Pinto, C. Gargioni, M.V. Alvarado-Mora, V.L. Pagliusi Castilho, et al., Detection of Schistosoma mansoni antibodies in a lowendemicity area using indirect immunofluorescence and circumoval precipitin test, Am. J. Trop. Med. Hyg. 90 (2014) 1146-1152.

[29] H.F. Kinkel, S. Dittrich, B. Baumer, T. Weitzel, Evaluation of eight serological tests for diagnosis of imported schistosomiasis, Clin. Vaccine Immunol. 19 (2012) 948-953.

[30] H. Smith, M. Doenhoff, C. Aitken, W. Bailey, M. Ji, et al., Comparison of Schistosoma mansoni soluble cercarial antigens and soluble egg antigens for serodiagnosing schistosome infections, PLoS Negl. Trop. Dis. 6 (2012) e1815.

[31] X. Xu, Y. Zhang, D. Lin, J. Zhang, J. Xu, et al., Serodiagnosis of Schistosoma japonicum infection: genome-wide identification of a protein marker, and assessment of its diagnostic validity in a field study in China, Lancet Infect. Dis. 14 (2014) 489-497.

[32] M. Berriman, B.J. Haas, P.T. LoVerde, R.A. Wilson, G.P. Dillon, et al., The genome of the blood fluke Schistosoma mansoni, Nature 460 (2009) 352-358.

[33] V. Silva-Moraes, J.M. Ferreira, P.M. Coelho, R.F. Grenfell, Biomarkers for schistosomiasis: towards an integrative view of the search for an effective diagnosis, Acta Trop. 132 (2014) 75-79.

[34] J.S. McCarthy, S. Lustigman, G.J. Yang, R.M. Barakat, H.H. Garcia, et al., A research agenda for helminth diseases of humans: diagnostics for control and elimination programmes, PLoS Negl. Trop. Dis. 6 (2012) e1601.

[35] G.B. Carvalho, L.G. Pacifico, D.L. Pimenta, L.M. Siqueira, A. Teixeira-Carvalho, et al., Evaluation of the use of C-terminal part of the Schistosoma mansoni $200 \mathrm{kDa}$ tegumental protein in schistosomiasis diagnosis and vaccine formulation, Exp. Parasitol. 139 (2014) 24-32.

[36] D.J. Kemp, R.L. Coppel, R.F. Anders, Repetitive proteins and genes of malaria, Annu. Rev. Microbiol. 41 (1987) 181-208.

[37] L. Schofield, On the function of repetitive domains in protein antigens of Plasmodium and other eukaryotic parasites, Parasitol. Today 7 (1991) 99-105.

[38] Y. Goto, R.N. Coler, S.G. Reed, Bioinformatic identification of tandem repeat antigens of the Leishmania donovani complex, Infect. Immun. 75 (2007) 846-851.

[39] Y. Goto, M.S. Duthie, S. Kawazu, N. Inoue, D. Carter, Biased cellular locations of tandem repeat antigens in African trypanosomes, Biochem. Biophys. Res. Commun. 405 (2011) 434-438.

[40] Y. Goto, D. Carter, S.G. Reed, Immunological dominance of Trypanosoma cruzi tandem repeat proteins, Infect. Immun. 76 (2008) 3967-3974.

[41] J.M. Angeles, Y. Goto, M. Kirinoki, L. Leonardo, P. Tongol-Rivera, et al., Human antibody response to thioredoxin peroxidase- 1 and tandem repeat proteins as immunodiagnostic antigen candidates for Schistosoma japonicum infection, Am. J. Trop. Med. Hyg. 85 (2011) 674-679.

[42] R.E. Davis, A.H. Davis, S.M. Carroll, A. Rajkovic, F.M. Rottman, Tandemly repeated exons encode 81-base repeats in multiple, developmentally regulated Schistosoma mansoni transcripts, Mol. Cell. Biol. 8 (1988) 4745-4755.

[43] J. Hamburger, I. Abbasi, C. Kariuki, A. Wanjala, E. Mzungu, et al., Evaluation of loopmediated isothermal amplification suitable for molecular monitoring of schistosome-infected snails in field laboratories, Am. J. Trop. Med. Hyg. 88 (2013) 344-351.

[44] I. Abbasi, C.H. King, E.M. Muchiri, J. Hamburger, Detection of Schistosoma mansoni and Schistosoma haematobium DNA by loop-mediated isothermal amplification: identification of infected snails from early prepatency, Am. J. Trop. Med. Hyg. 83 (2010) 427-432

[45] L. Cnops, P. Soentjens, J. Clerinx, M. Van Esbroeck, A Schistosoma haematobiumspecific real-time PCR for diagnosis of urogenital schistosomiasis in serum samples of international travelers and migrants, PLoS Negl. Trop. Dis. 7 (2013) e2413.

[46] H.M. Hussein, M.M. El-Tonsy, R.A. Tawfik, S.A. Ahmed, Experimental study for early diagnosis of prepatent schistosomiasis mansoni by detection of free circulating DNA in serum, Parasitol. Res. 111 (2012) 475-478.

[47] S.R. Smithers, R.J. Terry, The infection of laboratory hosts with cercariae of Schistosoma mansoni and the recovery of the adult worms, Parasitology 55 (1965) 695-700.

[48] F. Lewis, Schistosomiasis, Current Protocols in Immunology, John Wiley \& Sons, Inc., 2001

[49] K.P. Singh, H.C. Gerard, A.P. Hudson, D.L. Boros, Expression of matrix metalloproteinases and their inhibitors during the resorption of schistosome egg-induced fibrosis in praziquantel-treated mice, Immunology 111 (2004) 343-352.

[50] R.D. Isokpehi, O. Mahmud, A.N. Mbah, S.S. Simmons, L. Avelar, et al., Developmental regulation of genes encoding universal stress proteins in Schistosoma mansoni, Gene Regul. Syst. Biol. 5 (2011) 61-74.

[51] K. Okamoto, Y. Endo, S. Inoue, T. Nabeshima, P.T. Nga, et al., Development of a rapid and comprehensive proteomics-based arboviruses detection system, J. Virol. Methods 167 (2010) 31-36.

[52] F.J. Logan-Klumpler, N. De Silva, U. Boehme, M.B. Rogers, G. Velarde, et al. GeneDB-an annotation database for pathogens, Nucleic Acids Res. 40 (2012) D98-D108.

[53] G. Benson, Tandem repeats finder: a program to analyze DNA sequences, Nucleic Acids Res. 27 (1999) 573-580.

[54] R.P. Werkhauser, S.G. De Simone, E. Xavier, S.M. Montenegro, F.G. Abath, Mapping of the N terminus of the Schistosoma mansoni tegumental antigen Sm15 to its predicted precursor protein, Int. J. Parasitol. 30 (2000) 859-861.

[55] F.G. Abath, P. Hagan, S.A. Jeffs, A.J. Simpson, Partial characterization and kinetics of expression of Sm15, a Schistosoma mansoni tegumental antigen, Parasitol. Res. 80 (1994) 64-69.

[56] R.F. Grenfell, W.H. Martins, V. Silva-Moraes, S.V. Barata, E.G. Ribeiro, et al., Antigens of worms and eggs showed a differentiated detection of specific IgG according to the time of Schistosoma mansoni infection in mice, Rev. Soc. Bras. Med. Trop. 45 (2012) 505-509.

[57] R. Hussain, G. Dawood, N. Abrar, Z. Toossi, A. Minai, et al., Selective increases in antibody isotypes and immunoglobulin $\mathrm{G}$ subclass responses to secreted antigens in tuberculosis patients and healthy household contacts of the patients, Clin. Diagn. Lab. Immunol. 2 (1995) 726-732.

[58] M. Jiz, J.F. Friedman, T. Leenstra, B. Jarilla, A. Pablo, et al., Immunoglobulin E (IgE) responses to paramyosin predict resistance to reinfection with Schistosoma japonicum and are attenuated by IgG4 Infect. Immun. 77 (2009) 2051-2058.

[59] D. Negrao-Correa, J.F. Fittipaldi, J.R. Lambertucci, M.M. Teixeira, C.M. Antunes, et al., Association of Schistosoma mansoni-specific $\operatorname{IgG}$ and $\operatorname{IgE}$ antibody production and clinical schistosomiasis status in a rural area of Minas Gerais, Brazil, PLoS One 9 (2014) e88042. 
[60] A.S. DuVall, J.K. Fairley, L. Sutherland, A.L. Bustinduy, P.L. Mungai, et al., Development of a specimen-sparing multichannel bead assay to detect antiparasite IgG4 for the diagnosis of Schistosoma and Wuchereria infections on the coast of Kenya, Am. J. Trop. Med. Hyg. 90 (2014) 638-645.

[61] T.E. Wellems, R.J. Howard, Homologous genes encode two distinct histidine-rich proteins in a cloned isolate of Plasmodium falciparum, Proc. Natl. Acad. Sci. U. S. A. 83 (1986) 6065-6069.

[62] E.P. Rock, K. Marsh, A.J. Saul, T.E. Wellems, D.W. Taylor, et al., Comparative analysis of the Plasmodium falciparum histidine-rich proteins HRP-I, HRP-II and HRP-III in malaria parasites of diverse origin, Parasitology 95 (Pt 2) (1987) 209-227.
[63] D.P. Nolan, D.G. Jackson, H.J. Windle, A. Pays, M. Geuskens, et al., Characterization of a novel, stage-specific, invariant surface protein in Trypanosoma brucei containing an internal, serine-rich, repetitive motif, J. Biol. Chem. 272 (1997) 29212-29221.

[64] J.W. McBride, X.J. Yu, D.H. Walker, Glycosylation of homologous immunodominant proteins of Ehrlichia chaffeensis and Ehrlichia canis, Infect. Immun. 68 (2000) 13-18.

[65] B. Nickel, S. Sayasone, Y. Vonghachack, P. Odermatt, H. Marti, Schistosoma mansoni antigen detects Schistosoma mekongi infection, Acta Trop. 141 (2015) 310-314. 